\title{
Chemical probing suggests Redox-regulation of the carbonic anhydrase activity of mycobacterial Rv1284
}

Lisa Nienaber ${ }^{1 \dagger}$, Elysia Cave-Freeman ${ }^{1 \dagger}$, Megan Cross $^{1 \dagger}$, Lyndel Mason ${ }^{1}$, Ulla-Maja Bailey ${ }^{1}$, Parisa Amani ${ }^{1}$, Rohan Davis ${ }^{1,2}$, Paul Taylor ${ }^{3} \&$ Andreas Hofmann ${ }^{1,2,4}$

${ }^{1}$ From the Eskitis Institute, Griffith University, Nathan, Queensland, Australia

${ }^{2}$ Queensland Tropical Health Alliance, Smithfield, Australia

${ }^{3}$ School of Biological Sciences, The University of Edinburgh, Scotland, UK

${ }^{4}$ Faculty of Veterinary and Agricultural Sciences, The University of Melbourne, Parkville, Victoria, Australia

\section{Key words}

beta-carbonic anhydrase; enzyme modulation; $\mathrm{pH}$ homeostasis; Redox regulation

\section{Correspondence}

Andreas Hofmann, Structural Chemistry program, Eskitis Institute, Griffith University, N75 Don Young Road, Nathan, Qld 4111, Australia, Tel.: +61-7-3735-4425; Fax: +61-7-3735-6036;

Email: a.hofmann@griffith.edu.au

${ }^{\dagger}$ These authors made equal contributions to the work

\section{Database}

Atomic coordinates and structure factors have been deposited with the PDB, accession numbers 4yf4, 4yf5, 4yf6. 
The mycobacterial enzyme Rv1284 is a member of the $\beta$-carbonic anhydrase family that has been deemed essential for survival of the pathogen. The active site cavity of this dimeric protein is characterised by an exceptionally small volume and harbours a catalytic zinc ion coordinated by two cysteine and one histidine residue side chains.

Using the natural products polycarpine and emodin as chemical probes in crystallographic experiments and stopped-flow enzyme assays, we describe here that the catalytic activity can be reversibly inhibited by oxidation. Oxidative conditions lead to removal of one of the active site cysteine residues from the coordination sphere of the catalytic metal ion by engagement in a disulphide bond with another cysteine residue close by. The subsequent loss of the metal ion, which is supported by crystallographic analysis, may thus render the protein catalytically inactive. The oxidative inhibition of Rv1284 can be reversed by exposing the protein to reducing conditions. Since the physical size of the chemical probes used in this study substantially exceeds the active site volume, we hypothesised that these compounds exert their effects from a surface-bound location and identified tyrosine 120 as a critical residue for oxidative inactivation. These findings link conditions of oxidative stress to $\mathrm{pH}$ homeostasis of the pathogen. Since oxidative stress and acidification are defence mechanisms employed by the innate immune system of the host, we suggest that Rv1284 may be a component of the mycobacterial survival strategy.

\section{Introduction}

Carbonic anhydrases are metal-dependent enzymes, catalysing the reversible hydration of carbon dioxide to form the bicarbonate anion and a proton, thus being a pivotal enzyme class for carbon fixation and $\mathrm{pH}$ regulation in living organisms. Enzymes with carbonic anhydrase activity are separated into five different classes $(\alpha-\zeta)[1,2]$. With exception of the $\zeta$ carbonic anhydrases, which uses cadmium as an alternative metal co-factor [3], all other carbonic anhydrases are described as zinc-dependent. The seven human carbonic anhydrases belong to class $\alpha ; \beta$-carbonic anhydrases are found in algae, plants and bacteria [4], including mycobacteria. Among the three genes in the Mycobacterium tuberculosis genome that code for proteins with similarities to carbonic anhydrases, is Rv1284. The gene is required for optimal growth [5], and elevated levels of the gene product are found under starvation conditions which are used to model persistent bacteria [6].

Despite the common features shared by $\alpha^{-}$and $\beta$-carbonic anhydrases, such as the catalytic activity of $\mathrm{CO}_{2}$ hydration and the active site metal, there are also considerable structural differences. First, at the level of quaternary structure, $\beta$-carbonic anhydrases are oligomeric enzymes in solution; the fundamental structural unit is a dimer (see Figure 1a), but tetramers (Rv1284, Rv3588c) [7] and octamers (Pisum sativum $\beta$-carbonic anhydrase) [8] have also been observed. And second, there is a marked difference in the coordination of the active site metal. In mammalian carbonic anhydrases ( $\alpha$ class), the protein provides coordinating ligands to the active site zinc ion by means of three histidine side chains. In contrast, $\beta$-carbonic anhydrases provide one histidine and two cysteine residue side chains as metal-coordinating groups (Cys35, His88, Cys91 in Rv1284) in the active state. Further coordination sites on the metal ion are occupied by at least one water molecule, consistent with the proposed zinc-hydroxide mechanism of $\mathrm{CO}_{2}$ hydration. A blocked state of some $\beta$-carbonic anhydrases has been 
observed, whereby an aspartic acid residue (Asp37 in Rv1284) makes direct contact with the active site metal and thus completes the four-site coordination sphere without leaving access for water; a switch between both states has been proposed as a likely mechanism [8], and this is in agreement with experimentally obtained sigmoid enzyme activity curves [9] expected for mechanisms that switch between $\mathrm{T}$ - and R-states.

Oxidising agents such as reactive oxygen and nitrogen species form part of the innate immune response by a host when invaded by a pathogen. This includes toxic molecules such as $\mathrm{H}_{2} \mathrm{O}_{2}$, NO and $\mathrm{CO}$, but also molecular oxygen and acidic environmental $\mathrm{pH}$. In order to survive this assault, pathogens have developed sensing and inactivation mechanisms that protect against this response by the host. In the case of $M$. tuberculosis, the environmental Redox state is further an important factor [10-14] besides nutrient starvation [6] to trigger transition between its latent and active states. Despite the presence of mycobacterial enzymes that exert a protective function (such as superoxide dismutase, catalase, alkyl hydroperoxidase and peroxiredoxins), it has been reported that $M$. tuberculosis lacks the typical bacterial Redox sensors (including Salmonella OxyR; Rhizobium FixL; E. coli SoxR, fumarate/nitrate reduction regulator, ArcB; and Streptomyces RexA). Instead, the heme-conjugated histidine kinases DosS and DosT monitor the presence of oxidative ligands and regulate the DosR regulon [15, 16].

We recently proposed that $\beta$-carbonic anhydrases, in particular the mycobacterial enzymes Rv1284 and Rv3588c, may be targeted by non-classical inhibitors which cause inhibition of the enzymatic function, but do not bind in the active site of these enzymes [9]. During those efforts, we identified the previously reported cytotoxic marine natural product polycarpine, which possesses significant in vivo anti-tumour activity against P388 murine leukemia, and high inhibitory activity against reverse transcriptases from Raus sarcoma and avian myeloblastosis viruses in vitro and $\mathrm{Na}^{+}, \mathrm{K}^{+}$-ATPase isolated from rat brains [17-19]. The fact that polycarpine is a symmetrical disulphide and therefore a potentially rather reactive molecule made us dismiss the compound as a lead compound for inhibitor design, but use it as a probe molecule instead [20].

By chemical probing, we disocvered that the mycobacterial carbonic anhydrase Rv1284 shows Redox-dependent enzymatic activity and may thus be regulated by environmental Redox conditions. One of the cysteine residues (Cys35) coordinating the catalytic zinc ion in the active site can be transformed into a cystine with residue Cys61 under oxidising conditions. This leads to a loss of the catalytic metal ion and thus annihilation of the enzymatic activity of the protein. This oxidation may be initiated by air exposure (and thus also reactive species implicated in oxidative stress, such as $\mathrm{H}_{2} \mathrm{O}_{2}$ ), but also by peripheral ligands capable of undergoing Redox reactions, such as the natural products polycarpine and emodin. The highly cytotoxic marine natural product polycarpine [17-19] is a symmetrically substituted disulphide; its oxidative effects are thus $\mathrm{pH}$-dependent. Emodin is an polyhydroxylated anthraquinone that has been found in various plants such as Rhamnus purshiana, Aloe spp., and Rumex spp. as well as fungal sources such as Penicillium spp., Aspergillus spp., and Dermocybe sanguinea [21]. A variety of different biological activities has been reported for this secondary metabolite, and includes antimicrobial, anti-cancer and laxative effects [21]. Anthraquinones can easily be reduced to anthrahydroquinones and thus constitute oxidising agents of significant strength. 


\section{Results}

\section{Detection of polycarpine interaction with $\mathrm{His}_{6}-\mathrm{Rv} 1284$}

In order to discover non-classical inhibitors of $\beta$-carbonic anhydrases, we have established a programme, whereby an in-house compound library of pure natural compounds is screened against $\mathrm{His}_{6}-\mathrm{Rv} 1284$ in thermal denaturation assays as a first instance to discover ligands interacting with the target protein $[9,20]$. Based on the results from these differential scanning fluorimetry (DSF) assays, polycarpine was identified as a ligand interacting with $\mathrm{His}_{6}-\mathrm{Rv} 1284$, since it elicited a temperature shift of $\Delta T_{m}=-3.2 \mathrm{~K}$, compared to the thermal denaturation of the protein in the absence of ligand.

\section{Crystal structures of Rv1284 in the presence of polycarpine or hydrogen peroxide}

As reported previously [7], Rv1284 (PDB entry 1ylk) crystallises in the orthorhombic space group F222 with two dimers in the asymmetric unit which are related by a crystallographic 2fold axis. Dimer formation is due to intimate contacts between two monomers (Figure 1a). This is achieved mainly by extending the five-stranded central $\beta$-sheet of one monomer to a tenstranded $\beta$-sheet in the dimer. Furthermore, the $\mathrm{N}$-terminal region of one monomer, which comprises 24 amino acids and includes an $\alpha$-helix formed by residues Thr2-Phe18, is wrapped around the other monomer in a clamp-like fashion.

In an attempt to obtain ligand-bound crystal structures of mycobacterial carbonic anhydrase $\mathrm{His}_{6}-\mathrm{Rv}_{1284}$ with ligands selected based on results from the DSF screening, polycarpine was subjected to co-crystallisation experiments with the purified recombinant protein. Analysis of Xray crystal structures of crystals obtained under a variety of different conditions did not reveal electron density for a protein-bound ligand, and were otherwise highly similar to the structure of the apo-protein. However, multiple conformations of residues in and around the active site of Rv1284 were observed (see Figure 2). In crystals obtained in the presence of polycarpine at the acidic $\mathrm{pH}$ of 5.0 (dataset 4yf5), Cys35 was engaged in a disulphide bond with Cys61, a residue backing on to the active site. The occupancy of the cystine conformation at $\mathrm{pH} 5.0$ was quantitative, depleting the active site metal centre of an essential coordinating group. The coordination site previously filled by the Cys35 thiolate was now occupied by a water molecule in some instances (molecules $\mathrm{A}$ and $\mathrm{C}$ in 4yf5), but led to departure of the zinc ion in others (molecules B and D in 4yf5). The absence of the active site metal, concomitantly, resulted in a widening of the active site cleft, since the loop harbouring Cys91 shifted sidewards by about 1.5 $\AA$ (Figure 3). However, overall there were no significant structural changes observed when compared to the apo protein (PDB entry 1ylk), which is illustrated by the low rms deviations (4yf5_A/1ylk_A: $0.21 \AA$; 1 yf5_B/1ylk_B: $0.30 \AA$ ). At the mildly acidic pH of 6.4 (dataset 4yf4), Cys35 existed in two conformations, one coordinating the active site zinc ion $(60 \%)$ and another engaged in cystine formation with Cys61 $(40 \%)$. Here, Cys61 took two conformations, one engaged in disulphide bond formation, and one that was engaged in hydrogen-bonding to the backbone carbonyl of Leu86. When crystals of apo- $-\mathrm{His}_{6}-\mathrm{Rv} 1284$ obtained under very similar $\mathrm{pH}$ conditions were soaked for 10 min with $10 \mathrm{mM} \mathrm{H}_{2} \mathrm{O}_{2}$, the conformations observed in the active site of this crystal structure (dataset 4yf6) were essentially the same as for dataset 4yf4, with two alternate conformations for Cys35 and Cys61.

Whereas crystals obtained from $\mathrm{co}^{-}$crystallisation experiments were of good to very good 
quality, achieving diffraction resolutions of up to $1.8 \AA$, the peroxide-soaked crystals suffered from the treatment as reflected by the lesser resolution of $3.0 \AA$ (see Table 1). However, the electron density obtained after molecular replacement with the structure of the apo protein clearly indicated the presence of a (partial) disulphide bond between Cys35 and Cys61.

\section{Metal analysis of aged Rv1284 samples}

Metal analysis of Rv1284 samples with a minimum shelf time of two weeks was done for zinc, iron and manganese using ICP-AES. 0.56 equivalents of zinc, 0.02 equivalents of iron and no manganese were found, confirming earlier reports of only partial occupancy of the Rv1284 active site by zinc [22]. The zinc content determined by ICP-AES is in excellent agreement with the occupancies determined for the active site zinc ions in the crystal structures, which range from 0.55 to 0.60 .

\section{Redox-dependency of Rv1284 enzymatic activity}

The lack of significant enzymatic activity of $\mathrm{N}$-terminally His-tagged Rv1284 in $\mathrm{CO}_{2}$ hydration assays has previously been reported [7,9]. In the course of our drug discovery programme aiming at non-classical $\beta$-carbonic anhydrase inhibitors, we observed that only freshly prepared untagged Rv1284 samples showed the expected catalytic activity in $\mathrm{CO}_{2}$ hydration assays. Samples that were stored at $4^{\circ} \mathrm{C}$ for longer than two weeks showed diminished or entirely abolished enzymatic activity. However, when purifying recombinant Rv1284 using $\mathrm{N}_{2}$-saturated buffers, the shelf-life of active enzyme preparations was substantially enhanced, and a sample measured after a 10 week storage period at $4^{\circ} \mathrm{C}$ still retained its original catalytic activity.

We then tested whether enzymatic activity of aged Rv1284 samples could be recovered from inactive samples by exposing it to reducing conditions. An aliquot of an Rv1284 preparation (Figure 4a, black filled circles) that lost activity due to storage for more than two weeks (Figure 4a, black non-filled circles) was incubated with DTT for 48 hrs, and a buffer-only sample of the same volume was subjected to the same protocol and used as a control. Since DTT interferes with the $\mathrm{pH}$ indicator monitoring in the $\mathrm{CO}_{2}$ hydration assay, the reducing agent was removed by excessive washing of sample and control using ultraftiltration. The buffer-only control was tested in the assay to confirm the absence of any artificial effects. With the reductively re-activated sample of Rv1284 we observed enzyme activity at levels previously recorded for the freshly purified protein (Figure 4a, green circles).

Incubation of an enzymatically active sample of Rv1284 with polycarpine in solution, in our hands, did not result in significant inhibition of enzymatic activity. The conditions tested included incubation times of up to one week and basic $(\mathrm{pH}=8.5)$ as well as mildly acidic $(\mathrm{pH}=$ 6.5) buffers. We thus searched for organic molecules with known oxidative properties and similar structural extent as polycarpine. Since the physical extent of such molecules exceeds the space provided in the very small active site cavity of mycobacterial carbonic anhydrases, these molecules cannot enter the active site of the folded protein. The anthraquinone emodin was chosen, and when incubating an enzymatically active preparation of Rv1284 (Figure 4b, black circles) with $0.1 \mathrm{mM}$ emodin at $\mathrm{pH} 8.5$ for one week, the enzymatic activity was essentially abolished (Figure 4b, blue circles). After subjecting the inactivated sample to DTT incubation (same procedure as above), the enzymatic activity of the sample was restored (Figure 4b, green 
circles).

Based on these results, we decided to use emodin instead of polycarpine as a probe molecule in further enzyme activity assays. Since the crystallographic analysis identified Cys61 as the crucial residue in oxidative inactivation of Rv1284, the mutant Cys61Ser was generated to validate the role of this residue in the proposed mechanism. When tested in the $\mathrm{CO}_{2}$ hydration assays, the mutant Rv1284-C61S showed activity that was indistinguishable of that of wild-type protein (Figure 4c, black circles). Incubation of the mutant with emodin under the same conditions did not affect the enzymatic activity (Figure 4c, blue circles).

A consequence of the hypothesis that molecules of the extent of emodin and polycarpine can not migrate into the active site of mycobacterial carbonic anhydrases is the existence of a pathway by which two electrons can be withdrawn from the Cys35-Cys61 pair upon oxidation. Inspection of the crystal structure of Rv1284 suggested Tyr120 as a potential path (Figure 1b,c). This residue shields the active site with its catalytic zinc ion from the protein surface and points its side chain hydroxyl group into the active site, whereas its the backbone is surface-exposed. We therefore generated a Tyr120Leu mutant to assess the putative role of this residue. Figure $4 \mathrm{~d}$ (black circles) shows the enzymatic activity of Rv1284-Y120L which was very similar to that of the wild-type protein. After subjecting the mutant to emodin incubation under the same conditions as described above, no change in the enzymatic activity was observed (Figure $4 \mathrm{~d}$ blue circles), supporting the idea of Tyr120 being part of the electron pathway.

\section{Discussion}

Our ongoing efforts to discover non-classical inhibitors of mycobacterial carbonic anhydrases are based on screening natural product-inspired compound libraries by differential scanning fluorimetry as a first-line experiment to identify potential effectors for the $\beta$-carbonic anhydrases Rv1284 and Rv3588c. The cytotoxic marine natural product polycarpine was identified in these thermal stability assays using $\mathrm{His}_{6}-\mathrm{Rv} 1284$, and thus subjected to $\mathrm{co}^{-}$ crystallisation experiments using $\mathrm{His}_{6}-\mathrm{Rv} 1284$ and $\mathrm{His}_{6}-\mathrm{Rv} 3588 \mathrm{c}$. Protein crystallography support of drug discovery for mycobacterial carbonic anhydrases has been difficult, since, to our knowledge, organic molecules containing more than six atoms have not been observed in ligandbound protein crystal structures so far.

The crystal structures of purified bacterially expressed $\mathrm{His}_{6}-\mathrm{Rv} 1284$ under ambient conditions (i.e. without addition of oxidising or reducing agents) showed an active site conformation where a partially occupied catalytic metal site is coordinated by the three conserved active site residues of $\beta$-carbonic anhydrases (Cys35, His88 and Cys91 in Rv1284). The presence of Cys61 in the loop region between strand $\beta 2$ and helix $\alpha 3$ allows for an oxidised conformation of Cys35 by means of disulphide bond formation, since Cys61 backs on to the active site and both cysteine side chains can adopt cystine conformation by a simple rotation around the $\mathrm{C} \alpha-\mathrm{C} \beta$ bond. This oxidised state of the active site could be achieved by a Redox reaction with small molecules that can enter the constrained active site, such as $\mathrm{H}_{2} \mathrm{O}_{2}$ (dataset 4yf6). Intriguingly, the same effect could be elicited by Redox-active substrates that are highly unlikely to enter the active site, as observed here serendipitously with polycarpine (dataset 4yf4). Due to the size and extent of polycarpine, there is no obvious access route for this molecule to the active site of Rv1284 
without major conformational changes.

Since no substantial rearrangements of the protein fold were observed in the crystal structure obtained in the presence of polycarpine (dataset 4yf4), we conclude that the reduction of polycarpine occurs as a half-reaction at the protein surface, and its Redox potential should thus be $\mathrm{pH}$-dependent, since protons delivered by the bulk solvent will be consumed (Figure 5). This expected $\mathrm{pH}$-dependence is in agreement with the crystal structure obtained in the presence of polycarpine at low $\mathrm{pH}$ (dataset 4yf5), where the quantitative cystine formation was observed, that even led to loss of the catalytic metal ion.

These crystallographic results suggest that Rv1284 is an enzyme that is subject to oxidative inactivation. Indeed, when conducting $\mathrm{CO}_{2}$ hydration assays with purified bacterially expressed Rv1284, we found that samples with a shelf life of more than two weeks lost enzymatic activity (Figure 4a), which may be explained by oxidation due to air contact. The idea of oxygen-induced inactivation is further supported by the prolonged shelf-life of samples that had been subjected to protection with nitrogen gas during purification. The enzymatic activity of inactivated protein samples could be restored by exposing those samples to reducing conditions (Figure 4a), and the crucial role of Cys61, which we believed to render a dysfunctional active site upon disulphide bond formation with Cys35, is evident from the crystal structures (Figure 2) as well as enzyme assays using a Cys61Ser mutant (Figure 4c).

The incubation of Rv1284 with polycarpine in solution for up to one week did not substantially affect the enzymatic activity of the protein neither under acidic nor basic conditions. This was a rather unexpected observation, since the compound had an oxidising effect in the crystal structures (Figure 2). However, the polycarpine-modified crystals of Rv1284 were only obtained after $\sim_{3}$ months from co-crystallisation experiments. The long exposure time of protein and compound in the crystallisation experiments is thus substantially longer than in the experiments testing enzyme activity. We therefore sought to replace polycarpine with another probe compound that possesses strong oxidative properties and a sufficient size so as to not being able to migrate into the active site of Rv1284. Both criteria led us to choose emodin, which, as expected, affects Rv1284 enzymatic activity by means of reversible oxidative inactivation (Figure 4b).

If ligands such as polycarpine and emodin indeed modify the conformation in the active site from a surface location on the protein, there must be a path by which electrons are channelled into/out of the active site cysteine Cys35. Inspection of the crystal structure highlighted Tyr120 as a possible electron-conducting residue (Figure 1b,c). To test this hypothesis, we constructed the mutant Tyr120Leu which was expected to restrict electron channelling in this location and thus render the protein largely in-susceptible to oxidative inactivation by surface-bound effectors. Figure 4d illustrates results from enzyme activity assays with the mutant Tyr120Leu and validate the crucial role of tyrosine 120 in this context.

Whereas the amino acid sequence alignment of $\beta$-carbonic anhydrases suggested that other members of this enzyme family may potentially possess a similar Redox feature as Rv1284, a rigorous structure-based analysis showed that this is not the case (Figure 6). At present, there are three enzymes with known three-dimensional structures that possess a cysteine residue in the loop region between $\beta 2$ and $\alpha 3$ : Porphyridium purpureum (1ddz; Cys177, Cys203), Saccharomyces cerevisae Nce103 (3eyx ; Cys84) and Sordaria macrospora (4o1k; Cys85). 
However, none of these residues is located spatially close enough to engage in a cystine formation with the active site cysteine residue (Figure 6). Among the currently known $\beta^{-}$ carbonic anhydrases, Rv1284 from M. tuberculosis thus appears to be the only enzyme that engages this particular Redox-regulation of enzymatic activity.

\section{Conclusions}

The discovery that the enzymatic activity of Rv1284 is susceptible to Redox conditions invites speculations as to a physiological significance of this mechanism, linking oxidative stimuli to changes in the $\mathrm{pH}$ homeostasis of the pathogen. Oxidative stress and acidification are central components of the innate immune system by which the host responds to pathogens (for a review see [23]). There is thus a widely held view that acid and other host defences, such as reactive oxygen species and reactive nitrogen intermediates act in synergy. Since oxidative inactivation of Rv1284 interrupts the enzymatic hydration of $\mathrm{CO}_{2}$ and thus production of protons, the lowering of the $\mathrm{pH}$ within the radius of action of Rv1284 would be halted. The Redox regulation of Rv1284 may thus be a component of the mycobacterial survival strategy. Intriguingly, the molecular workings of this mechanism resembles what has been observed with the zinc-associated anti- $\sigma$ (ZAS) factor RslA. Mycobacterial RslA and the transcription factor $\sigma^{\mathrm{L}}$ are part of the transcription machinery that responds to oxidative stimuli. In RslA, residues from the CXXC motif can form a vicinal disulphide bond leading to dismissal of the bound zinc ion which in turn disengages RslA from $\sigma^{\mathrm{L}}$ due to conformational changes on RslA [24].

Furthermore, in the absence of any ligand-bound crystal structures of mycobacterial carbonic anhydrases with small-molecule compounds possessing more than six atoms, this study provides a first mapping of a ligand binding site on the surface of Rv1284, namely in an area around Tyr120.

\section{Materials and methods \\ Cloning and mutagenesis of expression constructs}

The cDNA construct of N-terminally $\mathrm{His}_{6}$-tagged Rv1284 in pCR-T7 [22] was a kind gift from TA Jones. This construct was used for subcloning of untagged Rv1284 into pRSET_6c [25] as described earlier [9]. Mutant constructs of Rv1284 were generated by the QuickChange ${ }^{\circledR}$ method, which is based on inverse PCR [26]. A pair of homologous primers (C61S mutation, coding: 5'-C AAC GCC GGA TCC GTG GTC ACC GAC GAT GTG ATC CGT TCA CTG-3’; C61S mutation, non-coding: 5'-GTC GGT GAC CAC GGA TCC GGC GTT GCG GAT GAC GTG TG-3'; Y120L mutation, coding: 5’-GG TCG CCC GA $A$ AGC TTG CCC GAC GCC GTC GAG GAC GTC CG-3'; Y120L mutation, non-coding: 5’-C GGC GTC GGG $\underline{\mathrm{C} A A} G C T$ TTC GGG CGA CCA CGT GGG TCT G-3’) carrying the mutations were designed for each mutant protein, and used in a PCR reaction performed by PfuUltra II Fusion HS DNA Polymerase (Agilent Technologies, Integrated Sciences Pty. Ltd., Preston, Australia). The PCR product was then treated with $D p n$ I to digest the parental DNA template prior to transformation into chemically competent Escherichia coli XL1Blue cells generated in house. Single colonies were picked, propagated and cDNA was purified using the NucleoBond Xtra kit (MN; Scientifix Life, Brisbane, Queensland, Australia). All constructs were validated by DNA sequencing using 
BigDye ${ }^{\mathrm{TM}}$ chemistry.

\section{Preparation of recombinant proteins}

Bacterially expressed recombinant $\mathrm{N}$-terminally $\mathrm{His}_{6}$-tagged Rv1284 as well as untagged Rv1284 were produced and purified as described earlier [9]. Briefly, the expression plasmids were transformed into Escherichia coli BL21-AI, and a total of 8 L of LB+ medium [27] and $50 \mu \mathrm{g} \mathrm{mL}$ ${ }^{1}$ ampicillin were inoculated with $1 / 10$ of its volume from an overnight liquid culture. The cells were grown at $37^{\circ} \mathrm{C}$ for $4 \mathrm{hrs}$ and then induced with either $0.2 \%$ arabinose $\left(\mathrm{His}_{6}-\mathrm{Rv} 1284\right.$ ) or $0.2 \%$ arabinose and $0.5 \mathrm{mM}$ IPTG (untagged Rv1284). After another $4 \mathrm{hrs}$ of incubation at $37^{\circ} \mathrm{C}$, cells were harvested, resuspended in $100 \mathrm{mM} \mathrm{NaCl}, 1 \mathrm{mM}$ EDTA, $20 \mathrm{mM}$ TRIS (pH 8), 0.1\% Triton $\mathrm{X}-100,1 \mathrm{mM}$ PMSF, $5 \mathrm{mM}$ benzamidinium chloride, and subsequently lysed by multiple freeze-thaw cycles and sonication. The cell lysate was subjected to ultracentrifugation $\left(100000 \times g, 45 \mathrm{~min}, 4^{\circ} \mathrm{C}\right)$ and proteins purified by either $\mathrm{Ni}^{2+}$ affinity chromatography $\left(\mathrm{His}_{6}{ }^{-}\right.$ Rv1284) or ion exchange chromatography (untagged Rv1284). The purified protein samples were concentrated by ultrafiltration. In the final cycles, the buffer was exchanged to $2 \%$ glycerol, 100 $\mathrm{mM} \mathrm{NaCl}, 20 \mathrm{mM}$ HEPES ( $\mathrm{pH}=7.5$ ). All purification steps were monitored by SDS-PAGE.

For $\mathrm{N}_{2}$-saturated buffers, MilliQ water was first degassed and then saturated with $\mathrm{N}_{2}$ by injecting a stream of $\mathrm{N}_{2}$ gas for 30 min. Working buffers were constituted from highly concentrated stock solutions of ingredients, diluted with $\mathrm{N}_{2}$-saturated water.

\section{Metal analysis}

Metal content analysis of bacterially expressed and purified $\operatorname{Rv} 1284\left(\rho *=1 \mathrm{mg} \mathrm{ml}^{-1}\right.$ ) was done by inductively coupled plasma atomic emission spectroscopy for zinc, manganese and iron using a using a Perkin Elmer Optima 8300 instrument. Calibration curves were obtained using serial dilutions of 1000 ppm standards (ICP-OES analytical standards, Perkin Elmer, Glen Waverley, Australia) for these metals.

\section{Chemicals}

The in-house natural product library used in this study consists of some 350 distinct and purified compounds, most of which have been obtained from Australian natural sources, and a small percentage $(\sim 5 \%)$ are known commercial drugs or synthetic compounds inspired by natural products. The library included the bis-TFA salt of polycarpine, which was isolated from several Polycarpa spp. collected on the Great Barrier Reef, as well as emodin (\#E7881) that was sourced from Sigma Aldrich (Castle Hill, Australia).

\section{Differential scanning fluorimetry}

The optimal ratio of protein and fluorescence dye was optimised by testing a $4 \times 5$ matrix of conditions varying the protein concentration from $2.5 \mu \mathrm{M}$ to $40 \mu \mathrm{M}$, and SYPRO Orange (Invitrogen; Life Technologies, Mulgrave, Victoria, Australia) concentration between $5 \times$ and $20 \times$, using a sample volume of $20 \mu \mathrm{l}$ with a buffer composed of $100 \mathrm{mM} \mathrm{NaCl}, 20 \mathrm{mM}$ HEPES (pH 7.5). For Rv1284, the best conditions were determined to contain $40 \mu \mathrm{M}$ protein and $6.5 \times$ SYPRO Orange. At least three technical replicas were then tested for for each ligand using the optimised protein-dye ratio. Ligands were added at a final concentration of $250 \mu \mathrm{M}$ in $20 \mu \mathrm{l}$ 
sample aliquots, with a final DMSO concentration of 5\%. Experiments were conducted on a Roche LightCycler 480, and analysed using the software DMAN [28]. $\Delta T_{m}$ values were calculated as difference between ligand and DMSO control experiments.

\section{Protein crystallography}

Crystals of $\mathrm{N}$-terminally $\mathrm{His}_{6}$-tagged Rv1284 were obtained from a variety of different conditions, all of which belonged to the orthorhombic space group F222. The crystallisation conditions of the different datasets are given in Table 1. All crystals used in this study have been obtained by the sitting drop vapour diffusion method with crystallisation experiments carried out at $16^{\circ} \mathrm{C}$.

$\mathrm{X}$-ray diffraction data were collected at the in-house diffractometer (Rigaku MicroMax007-HF; $\mathrm{R}$-Axis IV++ detector; Rigaku $\mathrm{X}$-stream cryo equipment) and at the Australian synchrotron beam line MX1 [29] under cryogenic conditions $(\mathrm{T}=100 \mathrm{~K})$. Data sets were indexed with XDS [30], and scaling, truncation and analysis were performed with programs from the CCP4 suite [31]. The orthorhombic crystal structures with four molecules per asymmetric unit were solved by molecular replacement using an Rv1284 dimer model of PDB accession number 1ylk [22]. Model building was performed with $\mathrm{O}$ [32] and Coot [33], and interspersed with computational refinement of atomic positions, individual B-factors and TLS refinement with two groups (chains A, B and chains C,D) using Phenix [34]. The occupancies of active site metal ions as well as residues with multiple conformations were also subjected to refinement. We note that in dataset 4yf4 a residual positive $\mathrm{F}_{\mathrm{o}}-\mathrm{F}_{\mathrm{c}}$ density remains in close spatial vicinity of Cys35-S $\gamma$, a site that is partially occupied by a water molecule when Cys35 is engaged in disulphide bond formation with Cys61. We assume that this water molecule coordinates the zinc ion in the active site in the absence of Cys35-S $\gamma$ and the metal ion is present in the active site. Neither averaged maps (calculated with Phenix), composite omit maps (calculated with CNS [35]), nor modelling the partially occupied water molecule with $\mathrm{Cl}^{-}$, resulted in a reasonable and more sophisticated model. The geometry of the final models was scrutinised using MolProbity tools as implemented in Phenix [34].

\section{Enzyme activity assays}

To analyse the $\mathrm{CO}_{2}$ hydration kinetics catalysed by Rv1284, a stopped flow assay with $\mathrm{pH}$ indicator readout was employed [36]. The absorbance change of $m$-cresol purple at a wavelength of $572 \mathrm{~nm}$ was monitored using a Bio-Logic SFM-100 MOS LED stopped flow instrument. $\mathrm{CO}_{2}$ was obtained from BOC, Australia, and all solutions were made with freshly filtered and degassed deionised water. A stock solution of polycarpine was prepared in DMSO at $60 \mathrm{mM}$ concentration, the stock of $60 \mathrm{mM} \mathrm{H} \mathrm{H}_{2}$ was prepared freshly as aqueous solution. For an individual experiment, the sample buffer contained $100 \mathrm{mM} \mathrm{Na}_{2} \mathrm{SO}_{4}, 25 \mathrm{mM}$ TAPS (pH 8.5), $50 \mu \mathrm{M} \mathrm{m-}$ cresol purple, $5 \mathrm{\mu M}$ protein and various additions as specified. The substrate buffer consisted of $\mathrm{CO}_{2}$-saturated water to achieve final concentrations between 2 and $17 \mathrm{mM}$. The reactions were followed for the first $60 \mathrm{sec}$, and at least five traces were analysed for each individual experiment to determine the initial rates of the reaction. 


\section{Reduction of protein samples}

Aliquots $\left(500 \mu \mathrm{l}\right.$ at $\left.18-24 \mathrm{mg} \mathrm{ml}^{-1}\right)$ of Rv1284 wild-type and mutant protein samples that had been subjected to enzyme activity assays were incubated with $0.1 \mathrm{mM}$ DTT for $48 \mathrm{hrs}$. A buffer sample of the same volume was also incubated with DTT at the same concentration and used as a control. DTT was then removed from all samples by successively washing $(10 \times)$ with degassed buffer in an ultrafiltration device.

\section{Bioinformatic methods}

A total of 40 curated $\beta$-carbonic anhydrase sequences were extracted from UniProtKB based on annotation search, and combined with additional sequences obtained from the PDB. Nonredundant secondary structure-based amino acid sequence alignments were generated using the software SBAL [37] and secondary structure information either predicted with PSIPRED [38] or extracted from PDB files [39].

\section{Acknowledgements}

We gratefully acknowledge the help of James Cameron (Griffith University) with the ICP-AES measurements, and useful discussions with Graeme Stevenson (Eskitis Institute). We have no conflict of interest to declare.

\section{Author contribution}

LN and ECF performed the enzyme assays. MC, LM and UMB cloned and expressed the proteins. $\mathrm{MC}$ and $\mathrm{AH}$ collected diffraction data, and $\mathrm{AH}$ solved the crystal structures. RAD provided natural products. PA, RAD, PT and AH analysed data. AH conceived the study and wrote the paper with critical input from all authors.

\section{Funding source}

Research in the laboratory of AH is funded by the National Health and Medical Research Council (NHMRC), the Australian Research Council (ARC) and the Rebecca L Cooper Medical Research Foundation. RD wishes to acknowledge the NHMRC (APP1024314) and ARC (LP120200339) for funding. Parts of this research were undertaken on the MX1 beam line at the Australian Synchrotron, Victoria, Australia. 


\section{References}

1. Tripp BC, Smith K \& Ferry JG (2001) Carbonic anhydrase: new insights for an ancient enzyme. J. Biol. Chem. 276, 48615-48618.

2. Zimmerman SA \& Ferry JG (2008) The beta and gamma classes of carbonic anhydrase. Curr. Pharm. Des. 14, 716-721.

3. Lane TW, Saito MA, George GN, Pickering IJ, Prince RC \& Morel FMM (2005) Biochemistry: a cadmium enzyme from a marine diatom. Nature 435, 42.

4. Rowlett RS (2010) Structure and catalytic mechanism of the beta-carbonic anhydrases. Biochim. Biophys. Acta 1804, 362-373.

5. Sassetti CM, Boyd DH \& Rubin EJ (2003) Genes required for mycobacterial growth defined by high density mutagenesis. Mol. Microbiol. 48, 77-84.

6. Betts JC, Lukey PT, Robb LC, McAdam RA \& Duncan K (2002) Evaluation of a nutrient starvation model of Mycobacterium tuberculosis persistence by gene and protein expression profiling. Mol. Microbiol. 43, 717-731.

7. Suarez Covarrubias A, Bergfors T, Jones TA \& Högbom M (2006) Structural mechanics of the $\mathrm{pH}$-dependent activity of beta-carbonic anhydrase from Mycobacterium tuberculosis. J. Biol. Chem. 281, 4993-4999.

8. Kimber MS \& Pai EF (2000) The active site architecture of Pisum sativum beta-carbonic anhydrase is a mirror image of that of alpha-carbonic anhydrases. EMBO J. 19, 14071418.

9. von Gnielinski N, Nienaber L, Mason L, Ellis S, Triccas JA, Davis RA \& Hofmann A (2014) Non-classical beta-carbonic anhydrase inhibitors - Towards novel anti-mycobacterials. MedChemComm 5, 1563-1566.

10. Wayne LG \& Sohaskey CD (2001) Nonreplicating persistence of mycobacterium tuberculosis. Annu. Rev. Microbiol. 55, 139-163.

11. Voskuil MI, Schnappinger D, Visconti KC, Harrell MI, Dolganov GM, Sherman DR \& Schoolnik GK (2003) Inhibition of respiration by nitric oxide induces a Mycobacterium tuberculosis dormancy program. J. Exp. Med. 198, 705-713.

12. Kumar A, Deshane JS, Crossman DK, Bolisetty S, Yan B, Kramnik I, Agarwal A \& Steyn AJC (2008) Heme oxygenase-1-derived carbon monoxide induces the Mycobacterium tuberculosis dormancy regulon. J. Biol. Chem. 283, 18032-18039.

13. Shiloh MU, Manzanillo P \& Cox JS (2008) Mycobacterium tuberculosis senses host-derived carbon monoxide during macrophage infection. Cell Host Microbe 3, 323-330.

14. Schnappinger D, Ehrt S, Voskuil MI, Liu Y, Mangan JA, Monahan IM, Dolganov G, Efron B, Butcher PD, Nathan C, et al. (2003) Transcriptional Adaptation of Mycobacterium tuberculosis within Macrophages: Insights into the Phagosomal Environment. J. Exp. Med. 198, 693-704.

15. Bhat SA, Singh N, Trivedi A, Kansal P, Gupta P \& Kumar A (2012) The mechanism of redox sensing in Mycobacterium tuberculosis. Free Radic. Biol. Med. 53, 1625-1641.

16. Chim N, Johnson PM \& Goulding CW (2014) Insights into redox sensing metalloproteins in Mycobacterium tuberculosis. J. Inorg. Biochem. 133, 118-126.

17. Abas SA, Hossain MB, van Der Helm D, Schmitz FJ, Laney M, Cabuslay R \& Schatzman RC (1996) Alkaloids from the Tunicate Polycarpa aurata from Chuuk Atoll. J. Org. Chem. 
61, 2709-2712.

18. Kang H \& Fenical W (1996) Polycarpine dihydrochloride: a cytotoxic dimeric disulfide alkaloid from the Indian Ocean ascidian Polycarpa clavata. Tetrahedron Lett. 37, 23692372.

19. Radchenko OS, Novikov VL, Willis RH, Murphy PT \& Elyakov GB (1997) Synthesis of polycarpine, a cytotoxic sulfur-containing alkaloid from the ascidian Polycarpa aurata, and related compounds. Tetrahedron Lett. 38, 3581-3584.

20. Hofmann A, Wang CK, Osman A \& Camp D (2010) Merging Structural Biology with Chemical Biology: Structural Chemistry at Eskitis. Struct. Chem. 21, 1117-1129.

21. Dictionary of natural products database (2015) Taylor \& Francis Group.

22. Suarez Covarrubias A, Larsson AM, Högbom M, Lindberg J, Bergfors T, Björkelid C, Mowbray SL, Unge T \& Jones TA (2005) Structure and function of carbonic anhydrases from Mycobacterium tuberculosis. J. Biol. Chem. 280, 18782-18789.

23. Ehrt S \& Schnappinger D (2009) Mycobacterial survival strategies in the phagosome: defence against host stresses. Cell Microbiol. 11, 1170-1178.

24. Thakur KG, Praveena T \& Gopal B (2010) Structural and biochemical bases for the redox sensitivity of Mycobacterium tuberculosis RslA. J. Mol. Biol. 397, 1199-1208.

25. Schoepfer R (1993) The pRSET family of T7 promoter expression vectors for Escherichia coli. Gene 124, 83-85.

26. Stratagene (2004) Quikchange ${ }^{\circledR}$ multi site-directed mutagenesis kit. Instruction Manual, Catalog \#200514, Revision \#113002e.

27. Hofmann A (2014) Methods in structural chemistry - a lab manual. Structural Chemistry Program, Eskitis Institute, Griffith University.

28. Wang CK, Weeratunga SK, Pacheco CM \& Hofmann A (2012) DMAN: a Java tool for analysis of multi-well differential scanning fluorimetry experiments. Bioinformatics 28, 439440.

29. McPhillips TM, McPhillips SE, Chiu HJ, Cohen AE, Deacon AM, Ellis PJ, Garman E, Gonzalez A, Sauter NK, Phizackerley RP, et al. (2002) Blu-Ice and the Distributed Control System: software for data acquisition and instrument control at macromolecular crystallography beamlines. J. Synchr. Rad. 9, 401-406.

30. Kabsch W (1993) Automatic processing of rotation diffraction data from crystals of initially unknown symmetry and cell constants. J. Appl. Cryst. 26, 795-800.

31. Collaborative Computational Project Number 4 (1994) The CCP4 suite: programs for protein crystallography. Acta Crystallogr. D 50, 760-763.

32. Jones TA, Zou JY, Cowan S \& Kjeldgaard M (1991) Improved methods for building protein models in electron density maps and location of errors in these models. Acta Crystallogr. A 47, 110-119.

33. Emsley P \& Cowton K (2004) Coot: Model-Building Tools for Molecular Graphics. Acta Crystallogr. D 60, 2126-2132.

34. Adams PD, Afonine PV, Bunkóczi G, Chen VB, Davis IW, Echols N, Headd JJ, Hung L, Kapral GJ, Grosse-Kunstleve RW, et al. (2010) PHENIX: a comprehensive Python-based system for macromolecular structure solution. Acta Crystallogr. D 66, 213-221.

35. Brünger AT, Adams PT, Clore GM, Delano WL, Gros P, Grosse-Kunstleve RW, Jiang JS, 
Kuszewski J, Nilges N, Pannu NS, et al. (1998) Crystallography and NMR system (CNS): A new software system for macromolecular structure determination. Acta Crystallogr. D 54, 905-921.

36. Khalifah RG (1971) The carbon dioxide hydration activity of carbonic anhydrase. J. Biol. Chem. 246, 2561-2573.

37. Wang CK, Broder U, Weeratunga SK, Gasser RB, Loukas A \& Hofmann A (2012) SBAL: a practical tool to generate and edit structure-based amino acid sequence alignments. Bioinformatics 28, 1026-1027.

38. Bryson K, McGuffin LJ, Marsden RL, Ward JJ, Sodhi JS \& Jones DT (2005) Protein structure prediction servers at University College London. Nucl. Acids Res. 33, W36W38.

39. Wang CK \& Hofmann A (2015) An integrated Java tool for generating amino acid sequence alignments with mapped secondary structure elements. 3Biotech 5, 87-92.

40. Brünger A (1992) Free R value: a novel statistical quantity for assessing the accuracy of crystal structures. Nature 355, 472-475.

41. DeLano W (2002) The PyMOL Molecular Graphics System. http://www.pymol.org.

42. Dolinsky TJ, Czodrowski P, Li H, Nielsen JE, Jensen JH, Klebe G \& Baker NA (2007) PDB2PQR: Expanding and upgrading automated preparation of biomolecular structures for molecular simulations. Nucleic Acids Res. 35, W522-525.

43. Wang CK, Broder U, Weeratunga SK, Gasser RB, Loukas A \& Hofmann A (2012) SBAL: a practical tool to generate and edit structure-based amino acid sequence alignments. Bioinformatics 28, 1026-1027.

44. Wang CK \& Hofmann A (2015) An integrated Java tool for generating amino acid sequence alignments with mapped secondary structure elements. 3Biotech 5, 87-92.

45. Bryson K, McGuffin LJ, Marsden RL, Ward JJ, Sodhi JS \& Jones DT (2005) Protein structure prediction servers at University College London. Nucl. Acids Res. 33, W36W38. 
Table 1

\begin{tabular}{|c|c|c|c|}
\hline Data set & $\begin{array}{c}\text { 4yf5 } \\
\mathrm{His}_{6}-\mathrm{Rv} 1284 \text { :polycarpine (pH 5.0) }\end{array}$ & $\begin{array}{c}\text { 4yf4 } \\
\mathrm{His}_{6}-\mathrm{Rv} 1284 \text { :polycarpine (pH 6.4) }\end{array}$ & $\begin{array}{c}4 y f 6 \\
\mathrm{His}_{6}-\mathrm{Rv} 1284: \mathrm{H}_{2} \mathrm{O}_{2}(\mathrm{pH} \text { 6.5) }\end{array}$ \\
\hline Crystallisation conditions & $\begin{array}{l}\text { 15\% PEG 400, } 0.1 \mathrm{M} \mathrm{NaH}_{2} \mathrm{PO}_{4} / \mathrm{Na} \\
\text { citrate, } \mathrm{pH}=5,10 \mathrm{mM} \text { polycarpine }\end{array}$ & $\begin{array}{l}0.10 \mathrm{M} \mathrm{K} / \mathrm{Na} \text { tartrate, } 0.10 \mathrm{M} \\
\mathrm{MES} / \mathrm{NaOH}, \mathrm{pH}=6.4,10 \mathrm{mM} \\
\text { polycarpine }\end{array}$ & $\begin{array}{l}0.2 \mathrm{M} \mathrm{NaF}, 20 \% \text { PEG } 3500,0.1 \\
\mathrm{M} \text { Bis-TRIS propane, pH=6.5; } \\
\text { soaked with } 10 \mathrm{mM} \mathrm{H}_{2} \mathrm{O}_{2} \text { for } 10 \\
\text { min }\end{array}$ \\
\hline
\end{tabular}

\section{Data collection}

\begin{tabular}{|c|c|c|c|}
\hline X-ray source & AS MX1 & AS MX1 & AS MX1 \\
\hline Detector & ADSC Quantum 210r & ADSC Quantum 210r & ADSC Quantum 210r \\
\hline Wavelength $(\AA)$ & 0.95375 & 0.95382 & 0.95375 \\
\hline Space group & F222 & F222 & F222 \\
\hline Cell dimensions $(\AA)$ & $103.3,153.9,159.1$ & $101.1,154.4,157.7$ & $101.1,153.9,157.2$ \\
\hline Max. resolution $(\AA)$ & 2.0 & 1.8 & 3.0 \\
\hline$\langle\langle I\rangle /\langle\sigma(I)\rangle\rangle$ & $22.6(5.1)$ & $15.0(1.8)$ & $14.2(4.9)$ \\
\hline Wilson B-factor $\left(\AA^{2}\right)$ & 27.6 & 22.2 & 47.9 \\
\hline No of unique reflections & $42879(5941)$ & $56222(7594)$ & $12412(1777)$ \\
\hline Multiplicity & $13.7(12.7)$ & $5.4(3.1)$ & $14.9(14.6)$ \\
\hline Completeness & $0.992(0.949)$ & $0.988(0.924)$ & $0.997(0.994)$ \\
\hline $\mathrm{R}_{\mathrm{sym}}{ }^{\mathrm{a}}$ & $0.074(0.459)$ & $0.06(0.448)$ & $0.207(0.581)$ \\
\hline
\end{tabular}




\section{Refinement}

No of reflections in working / test 39382 / 2062

set

Visible residues

5084

$1-163$

249

No of water molecules

$4\left(2 \times \mathrm{Zn}^{2+}, 2 \times \mathrm{Cl}^{-}\right)$

No of ions

29.8

28.6

31.4

Ions

rmsd B-factor for bonded atoms 4.96

$\left(\AA^{2}\right)$

rmsd bond lengths $(\AA)$

0.008

rmsd bond angles $\left(^{\circ}\right)$

1.113

MolProbity analysis

Ramachandran favoured (\%) 96.7

Ramachandran allowed (\%) $\quad 3.26$

Ramachandran outliers (\%) 0

Rotamer outliers (\%) 3.55

C-beta outliers 0

Clashscore $\quad 5.13$

Overall score $\quad 1.93$

$\mathrm{R}-$ factor $^{\mathrm{b}}$

$0.197(0.268)$

$0.260(0.313)$
$49462 / 2669$

$11220 / 577$

5108

5108

$1-163$

$1-163$

321

$10\left(4 \times \mathrm{Zn}^{2+}, 4 \times \mathrm{Mg}^{2+}, 2 \times \mathrm{Cl}^{-}\right) \quad 8\left(4 \times \mathrm{Zn}^{2+}, 2 \times \mathrm{Mg}^{2+}, 2 \times \mathrm{Cl}^{-}\right)$

24.4

$28.0-32.4$

$27.5 \quad 37.0$

$4.30 \quad 8.40$

$0.008 \quad 0.009$

1.096

$99.7 \quad 94.0$

$0.31 \quad 5.40$

0

$\begin{array}{ll}4.6 & 7.7\end{array}$

$0-0$

$3.33 \quad 8.14$

$1.58-2.52$

$0.194(0.305) \quad 0.167(0.268)$

$0.244(0.324) \quad 0.260(0.356)$ 
Values in parentheses refer to the last resolution shell.

${ }^{\mathrm{a}} \mathrm{R}_{\text {sym }}=\Sigma \mid I-\langle\Lambda| / \Sigma I$, where $I$ is the observed intensity, and $\langle I\rangle$ is the average intensity obtained from multiple observations of symmetry-related reflections after rejections.

${ }^{\mathrm{b}} \mathrm{R}$-factor $=\Sigma|| F_{o}|-| F_{c}|/ \Sigma| F_{o} \mid$, where $F_{o}$ and $F_{c}$ are the observed and calculated structure factors, respectively.

${ }^{c} R_{\text {free }}$ defined 


\section{Figure Legends}

\section{Figure 1}

Overview of structural features of Rv1284.

$\boldsymbol{a}$ - Rv1284 forms an intimate homodimer. The two monomers (dataset 4yf4) are shown as cartoon representation and coloured in blue and green, respectively. The active site residues as well as Tyr120 of one monomer (blue) are drawn explicitly as stick models; the active site zinc ion is shown as magenta sphere. For the second monomer (green), the location of the active site is indicated by the rendered zinc ion. $b$ - A close-up of the active site of the blue monomer shows the relative orientation of Tyr 120 with respect to the active site residues involved in zinc coordination. $c$ - This surface representation (the colour gradient indicates the electrostatic potential: blue - positive, red - negative) shows the same view of the monomer as in $b$ and indicates the likely binding site of the chemical probes used in this study. Images were rendered with PyMOL [41], and the electrostatic potential was calculated using PDB2PQR and APBS [42].

\section{Figure 2}

The conformation in the active site of Rv1284 depends on the Redox state.

Stereo figures with $2 F_{o}-F_{c}$ electron density (contoured at $1 \sigma$ ) show the active sites of of three crystal structures: $\boldsymbol{a}-\mathrm{His}_{6}-\mathrm{Rv} 1284$ :polycarpine at $\mathrm{pH} 5.0$ (dataset $4 \mathrm{yf5}$ ); $\boldsymbol{b}-\mathrm{His}_{6}{ }^{-}$ Rv1284:polycarpine at pH 6.4 (dataset 4yf4); $c$ - $\mathrm{His}_{6}-\mathrm{Rv}_{2} 284: \mathrm{H}_{2} \mathrm{O}_{2}$ at pH 6.5 (dataset 4yf6). The numbers indicate the occupancies of the individual atoms or conformations. Zinc is shown as magenta and water as red spheres. Images were rendered with PyMOL [41].

\section{Figure 3}

Oxidation of Rv1284 can lead to loss of the catalytic zinc ion, which results in further conformational changes in the active site.

Shown is a superposition of molecule B of the crystal structures obtained from $\mathrm{His}_{6}{ }^{-}$ Rv1284:polycarpine at pH 5.0 (dataset 4yf5; green) and $\mathrm{His}_{6}-\mathrm{Rv} 1284$ :polycarpine at pH 6.4 (dataset 4yf4; blue). Oxidation of Cys35 by polycarpine at acidic $\mathrm{pH}$ is quantitative and can result of the departure of zinc from the active site due to unsatisfying coordination. This leads to a widening of the active site cleft as the loop harbouring Cys91 shifts sidewards. Figure rendered with PyMOL [41].

\section{Figure 4}

Stopped-flow measurements assessing the catalytic $\mathrm{CO}_{2}$ hydration activity by Rv1284 proteins. a - Oxidation of Rv1284 during storage results in loss of the $\mathrm{CO}_{2}$ hydration activity, which can be restored by addition of reducing agents. Michaelis-Menten profiles of the enzymatic $\mathrm{CO}_{2}$ hydration activity of purified recombinant Rv1284 were acquired using freshly purified protein (black filled circles, “active”), the same sample after 2 weeks' storage (black non-filled circles, "inactive") and inactive protein after treatment with DTT (green filled circles, "re-activated").

b - Oxidation of Rv1284 with $0.1 \mathrm{mM}$ emodin at $\mathrm{pH} 8.5$ for one week results in loss of the $\mathrm{CO}_{2}$ hydration activity, which can be restored by addition of reducing agents. Michaelis-Menten 
profiles of the enzymatic $\mathrm{CO}_{2}$ hydration activity of freshly purified recombinant Rv1284 (black filled circles) and after incubation with emodin (blue non-filled circles). Incubation of the emodintreated sample (same conditions as in a) with DTT restores enzymatic activity (green filled circles).

c - Cysteine-61 is critical for the Redox-dependent regulation of Rv1284 enzymatic activity. The mutant Rv1284-C61S displays $\mathrm{CO}_{2}$ hydration activity similar to the wild-type protein (black filled circles). Incubation of Rv1284-C61S with emodin (same conditions as in a) does not affect the enzymatic activity of the protein (blue non-filled circles).

d - The electron transfer pathway involves tyrosine-120. The mutant Rv1284-Y120L displays $\mathrm{CO}_{2}$ hydration activity similar to the wild-type protein (black filled circles). No significant effect on the enzymatic activity of the mutant is observed after incubation of Rv1284-Y120L with emodin (blue non-filled circles; same conditions as in a).

\section{Figure 5}

Structures of the chemical probes polycarpine and emodin, and their Redox reaction with mycobacterial Rv1284.

Polycarpine is a symmetrical disulphide that can be reduced by withdrawing electrons from Rv1284 whereby Cys35 and Cys61 undergo an oxidation to form a cystine. The reduction of polycarpine is $\mathrm{pH}$-dependent and the reduced form is favoured at lower $\mathrm{pH}$. Therefore, formation of the Cys35-Cys61 cystine is enhanced at acidic $\mathrm{pH}$, in agreement with the observation made in crystal structures obtained at pH 6.4 (dataset 4yf4) and pH 5.0 (dataset 4yf5).

The anthraquinone emodin is a powerful oxidation agent, since it can readily be reduced to an anthrahydroquinone. In this study, emodin was used to oxidise Rv1284 in solution.

\section{Figure 6}

Excerpt from a structure-based amino acid sequence alignment of $\beta$-carbonic anhydrases generated with SBAL from PDB files $[43,44]$ or PSIPRED [45] secondary structure predictions using amino acid sequences.

The amino acid sequence alignment from the superimposed three-dimensional structures of $\beta^{-}$ carbonic anhydrases shows that the cysteine residue in the active site is too distant from the cysteine residue in the loop region between $\beta 2$ and $\alpha 3$ in order to engage in cystine formation. Only in the case of Rv1284, Cys61 is spatially close enough to form a covalent bond. 